\title{
Warty cutaneous tuberculosis of the nose: A rare localization
}

\section{Moussa Doulla', Laouali Salissou', Nina Korsaga/Some', Maimouna Mamadou Ouedraogo', Larabou Aminou ${ }^{3}$, Mahamadou Zaki Harouna ${ }^{4}$, Soumana Alphazazi ${ }^{5}$, Pascal Niamba ${ }^{6}$, Adama Traoré ${ }^{6}$}

\begin{abstract}
${ }^{1}$ Department of Dermatology, National Hospital Niamey, Niamey, Niger, ${ }^{2}$ Department of Dermatology-Venereology of the CMA of Pissy Ouagadougou, Burkina Faso, ${ }^{3}$ Department of Dermatology-Venereology of the Reference Hospital of Maradi, Niger. ${ }^{4}$ Amirou Boubacar Diallo National Hospital of Niamey, Niger, ${ }^{5}$ National Center for the fight against Tuberculosis and Lung Diseases Niamey, Niger, ${ }^{6}$ Department of Dermatology-Venereology of CHU-YO, Ouagadougou, Burkina Faso
\end{abstract}

Corresponding author: Laouali Salissou, MD, E-mail: danmata@yahoo.com

\begin{abstract}
Cutaneous tuberculosis is a rare, extra-pulmonary form of tuberculosis caused by mycobacteria of the tuberculosis complex. It is characterized by clinical polymorphism often posing a difficult diagnostic challenge. Herein, we report a case of cutaneous tuberculosis in its warty form located on the nose. This was a 57 -year-old patient who was infected in the classroom three months previously while taking lessons from a woman with pulmonary tuberculosis. A facial examination revealed a blackish, papillomatous patch invading almost the entire nose, with a keratotic surface spreading over the wings of the nose. The diagnosis of verrucous tuberculosis was reached on the basis of epidemiological, clinical, and paraclinical arguments. Under anti-tuberculosis treatment for six months, the lesion had healed without sequelae. The diagnosis of verrucous cutaneous tuberculosis must be established in the presence of any chronic and crusty lesion. The management responds to the treatment protocol for all forms of tuberculosis.
\end{abstract}

Key words: Cutaneous tuberculosis; Warty; nose; Niamey; Niger

\section{INTRODUCTION}

Cutaneous tuberculosis includes all cutaneous manifestations due to mycobacteria of the tuberculosis complex: Mycobacterium tuberculosis, Mycobacterium bovis, and Mycobacterium africanum [1]. It is rare, representing $2 \%$ of cases of extrapulmonary tuberculosis [2-4]. Its clinical forms are highly numerous and much more poorly understood than in the past $[5,6]$. Diagnosis is often difficult due to the polymorphism of both clinical and histological pictures $[3,4,6,7]$. Herein, we report a case of cutaneous tuberculosis in its warty form located on the nose.

\section{CASE REPORT}

This was a 57-year-old housewife who consulted on March 10, 2018, for a keratotic lesion located on the nose and evolving for three months. A notion of contagion with a case of pulmonary tuberculosis was found during interrogation as well as a notion of evening fever and weight loss. A treatment combining metronidazole at a dose of $2 \mathrm{~g} /$ day per os for one month and traditional herbal medicine by local application had been instituted without success. On physical examination, the patient was in good general condition. A dermatological examination revealed a blackish, keratotic patch with a crusty, melliceric surface covering the entire nasal mass with an overflow in the nasolabial folds (Fig. 1). This lesion was neither itchy nor painful. In front of this case, we evoked cutaneous tuberculosis, leishmaniasis, basal cell carcinoma, and Kaposi's disease.

A skin biopsy with a pathological examination showed an inflammatory tuberculoid granuloma

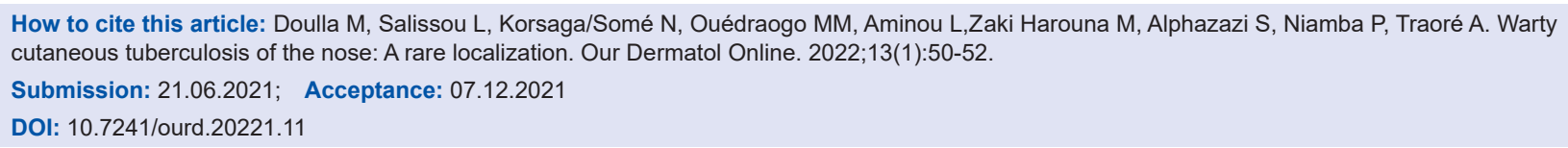




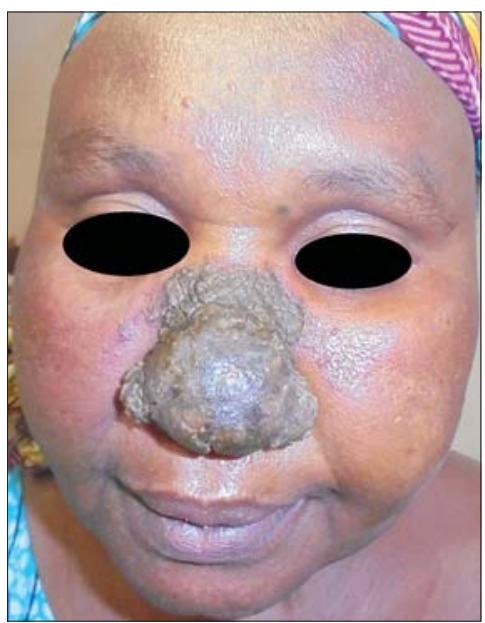

Figure 1: Before treatment (blackish patch with a keratotic surface invading almost the entire nose).

without caseous necrosis. Intradermal reaction to tuberculin (IDR) was negative. Dermal smear showed a Leishman body. Bacteriological and mycological examinations were negative as well. Hematologically, the sedimentation rate was elevated to $79 \mathrm{~mm}$ in the first hour and the blood count was normal. Blood sugar and the exploration of kidney function were also normal. Retroviral serology was negative. An AP chest X-ray was normal (Fig. 2). The diagnosis of warty cutaneous tuberculosis was retained on the basis of epidemiological, clinical, and paraclinical arguments. The patient was started on anti-tuberculosis treatment according to the protocol for the treatment of all forms of tuberculosis. It is first quadruple therapy (isoniazid at $3-5 \mathrm{mg} / \mathrm{kg} / \mathrm{d}$, rifampicin at $10 \mathrm{mg} / \mathrm{kg} / \mathrm{d}$, ethambutol at 15-20 mg/kg/d, and pyrazinamide at 20-30 mg/kg/d) for two months, then dual therapy (isoniazid at $300 \mathrm{mg} / \mathrm{d}$ and rifampicin at $400 \mathrm{mg} / \mathrm{d}$ ) for four months. Local treatment with a keratolytic (3\% salicylated petroleum jelly) and fusidic acid was included. Significant lysis of the keratosis was noted at month three of treatment (Fig. 3), and clinical healing without sequelae was noted at month six of treatment with a two-year follow-up (Fig. 4).

\section{DISCUSSION}

Warty cutaneous tuberculosis results from skin re-inoculation of Koch's bacillus in a previously sensitized subject [5,7-9], yet this notion of reinoculation was not reported in our patient. As in the case of our observation, a lesion most often results in one or more papillomatous, keratotic, painless patches of progressive extension $[5,10]$. Unlike our case, in

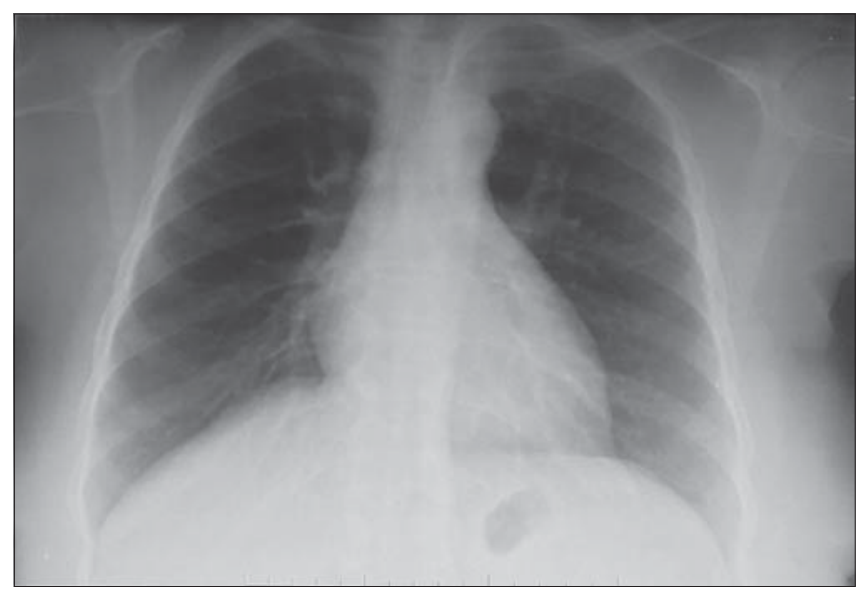

Figure 2: Frontal chest X-ray (normal X-ray).

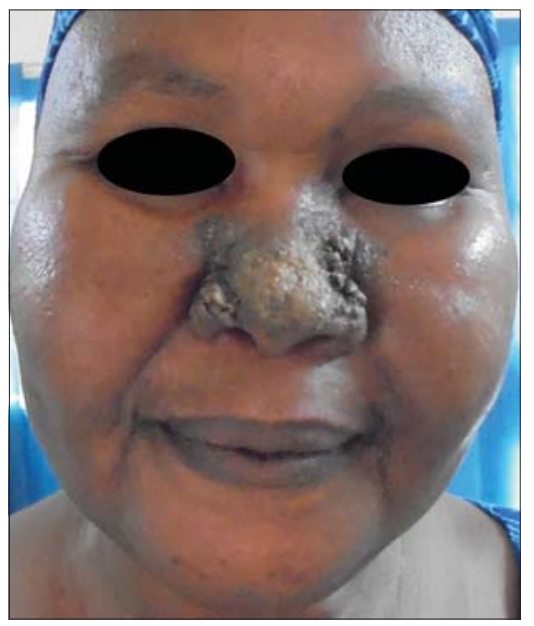

Figure 3: Evolution after three months of treatment (lysis and stripping of keratosis).

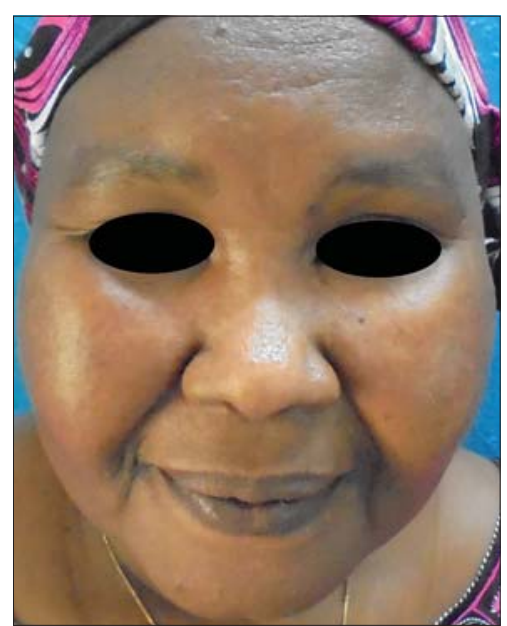

Figure 4: End of treatment (complete disappearance of the lesions after six months of treatment).

which the topography was nasal, a lesion is preferentially located in the extremities, especially in the hands and face, yet also in the perianal region $[5,7,10]$, and may 
sometimes be associated with lymphadenopathy $[5,8]$. However, a case of warty cutaneous tuberculosis of a nasal localization was reported in a study by Chaabane et al. [11]. As in our patient, these types of lesions are clinically suggestive of a verrucous squamous cell carcinoma, cutaneous leishmaniasis, or typical or atypical mycobacterial infections $[8,12]$. According to the literature, the anatomo-pathological examination of a biopsy piece often reveals caseous necrosis $[5,8]$, which was not found in our case, as reported by Chaabane et al. [11].

The tuberculin skin test was also positive in several studies $[5,8]$. It was negative in our patient. Cultures are most often negative, making the diagnosis difficult $[5,10]$. It is in such a situation that gene amplification by PCR is highly necessary [12], which was not conducted in our case due to the lack of a technical platform. The diagnosis of warty cutaneous tuberculosis was retained in a combination of the following arguments: the notion of contagion, the appearance of the lesion, the elevation of the SV, the aspect of the tuberculoid granuloma without caseous necrosis on histology, the unsuccessful evolution following previous treatment (metronidazole at $2 \mathrm{~g} / \mathrm{d}$ ), despite the negativity of the rest of the assessment. In our patient, the recovery obtained with anti-tuberculosis treatment retrospectively constituted the strongest argument in favor of the diagnosis of tuberculosis, as in several other studies $[12,13]$.

\section{CONCLUSION}

Warty cutaneous tuberculosis, despite its rarity, should be considered in the presence of any chronic skin disease resistant to conventional local treatment, because of its polymorphism. The difficulty of obtaining bacteriological confirmation in our context (direct examination, culture, polymerase chain reaction (PCR)) requires collecting a conjunction of anamnestic, clinical, and histological arguments.

The absence of caseous necrosis on histopathological examination should not exclude the diagnosis, as it is most often found in multiple non-specific cases with infiltrates of various compositions and arrangements. A well-conducted treatment with anti-tuberculosis drugs without resistance usually progresses toward healing, sometimes without sequelae. Such a response to a specific anti-tuberculosis treatment constitutes a major diagnostic argument.

\section{Consent}

The examination of the patient was conducted according to the principles of the Declaration of Helsinki.

The authors certify that they have obtained all appropriate patient consent forms, in which the patient her consent for images and other clinical information to be included in the journal. The patients understand that their names and initials will not be published and due effort will be made to conceal their identity, but that anonymity cannot be guaranteed.

\section{REFERENCES}

1. Dicko A, Faye O, Fofana Y, Soumoutera M, Berthé S, Touré, et al. [Cutaneous tuberculosis in Bamako, Mali]. Pan Afr Med J. 2017;27:102.

2. Fekih N El, Fazaa B, Kerkeni N, Sfia M, Zeglaoui F, Zermani R, et al. Tuberculous lupus. Med Mal Infect. 2009;39:409-12.

3. Bilan P, Sin C, Wann AR, Grossin M, Courdavault L, Sigal ML, et al. Cutaneous tuberculosis and erythema induratum: A retrospective study of 13 cases in France. Ann Dermatol Venereol. 2015;142:237-44.

4. Losada-López I, García-Gasalla M, Taberner R, Cifuentes-Luna C, Arquinio L, Terrasa F, et al. Cutaneous tuberculosis in an area of Mallorca (2003-2011). Rev Clin Esp. 2012;212:179-83.

5. Tigoulet F, Fournier V, Caumes E. Clinical forms of the cutaneous tuberculosis. Bull Soc Pathol Exot. 2003;96:362-67.

6. Salissou L, Adehossi E, Maman Laouali S, Mamadou S, Nouhou H. Cutaneous tuberculosis in Niger: A 9-year retrospective study. Our Dermatol Online. 2015;6:153-56.

7. Diop. A, Ndiaye MT, Ndiaye M, Seck B, Diouf A, Diatta BH, et al. Rare cutaneous tuberculosis in sub-Saharan Africa developed on discoid lupus erythematous lesion. Bull Soc Pathol Exot. 2017;110:230-3.

8. Hamada S, Benhiba H, Benzekri L, Senouci K, Hassam B. Kissing lesions heralding vegetative tuberculosis. Ann Dermatol Vénéréol. 2013;140:67-8.

9. Agarwala N, Mohapatra M, Hassanandani T, Panda M. Coexistence of tuberculous gumma with tuberculosis verrucous cutis (TBVC) in an immunocompetent female. Our Dermatol Online. 2020;11:62-4.

10. Morand JJ, Lightburn E. Cutaneous tuberculosis. EMC (Elsevier Masson SAS, Paris) Dermatologie 98-360-A-10, 2007:14p.

11. Chaabane H, Chami I, Charfi S, Masmoudi A, Amouri M, Makni S, et al. Lesions of the nose revealing warty tuberculosis. Press Med. 2014;44:372-3.

12. Attallah S, Hali F, Chaibi Y, Marnissi F, Benchikhi H. Lupus vulgaris tumor nickname of the forearm. Ann Dermatol Vénéréol. 2012;139:B235-B236.

13. Amouri M, Ben Salah R, Chaaben H, Meziou TJ, Mseddi M, Turki H. Chronic tubercular lupus. Ann Dermatol Vénéréol. 2011;138:637-8.

Copyright by Moussa Doulla, et al. This is an open-access article distributed under the terms of the Creative Commons Attribution License, which permits unrestricted use, distribution, and reproduction in any medium, provided the original author and source are credited.

Source of Support: Nil, Conflict of Interest: None declared. 